\title{
Open system dynamics of simple collision models *
}

\author{
Mário Ziman ${ }^{1,2}$ and Vladimír Bužek ${ }^{1,2}$ \\ ${ }^{1}$ Institute of Physics, Slovak Academy of Sciences, Dúbravská cesta 9, \\ 84511 Bratislava, Slovakia, \\ ${ }^{2}$ Faculty of Informatics, Masaryk University, Botanická 68 a \\ 602 00 Brno, Czech Republic
}

\begin{abstract}
A simple collision model is employed to introduce elementary concepts of open system dynamics of quantum systems. In particular, within the framework of collision models we introduce the quantum analogue of thermalization process called quantum homogenization and simulate quantum decoherence processes. These dynamics are driven by partial swaps and controlled unitary collisions, respectively. We show that collision models can be used to prepare multipartite entangled states. Partial swap dynamics generates $W$-type of entanglement saturating the CKW inequalities, whereas the decoherence collision models creates $G H Z$-type of entangled states. The considered evolution of a system in a sequence of collisions is described by a discrete semigroup $\mathcal{E}_{1}, \ldots, \mathcal{E}_{n}$. Interpolating this discrete points within the set of quantum channels we derive for both processes the corresponding Linblad master equations. In particular, we argue that collision models can be used as simulators of arbitrary Markovian dynamics, however, the inverse is not true.
\end{abstract}

\section{Open system dynamics}

The goal of these lectures is to present the elementary ideas and features of dynamics of open quantum systems by analyzing the properties of the simplest collision model we can think of. The material is based on papers [1, 2, 3, 4, on collision models coauthored by authors. It is not considered as a review paper on collision models or open system dynamics.

It turns out it is surprisingly useful in physics to distinguish the concepts of isolated and open systems. The concept of isolated systems represents a simplification of physical reality, which serves as a playground for a clear formulation

\footnotetext{
${ }^{*}$ Lecture presented at 46 Karpacz Winter School of Theoretical Physics Ladek Zdroj, Poland, 8 - 13 February 2010
} 
of elementary physical principles. It is postulated that the dynamics of isolated quantum systems is driven by Schrödinger equation [5]

$$
i \hbar \frac{d}{d t} \psi_{t}=H_{t} \psi_{t},
$$

where $H_{t}$ is a hermitian operator called Hamiltonian. As a consequence it follows that the state transformations are unitary, i.e. $\psi_{t} \rightarrow \psi_{t^{\prime}}=U_{t \rightarrow t^{\prime}} \psi_{t}$, where $U_{t \rightarrow t^{\prime}} U_{t \rightarrow t^{\prime}}^{\dagger}=U_{t \rightarrow t^{\prime}}^{\dagger} U_{t \rightarrow t^{\prime}}=I$. If $H_{t}$ is time-independent, i.e. $H_{t} \equiv H$, then $U_{t \rightarrow t^{\prime}}=\exp \left[-\frac{i}{\hbar} H\left(t^{\prime}-t\right)\right]$. We can define $U_{t}=\exp \left(-\frac{i}{\hbar} H t\right)$ and write $U_{t \rightarrow t^{\prime}}=U_{t^{\prime}} U_{t}^{\dagger}, \psi_{t}=U_{t} \psi_{0}$.

Interactions between a system under consideration and its environment are responsible for the violation of system's (dynamical) isolation. The joint evolution of the system and the environment will be still unitary, however, the system itself undergoes a different dynamics. The point is that these interactions, because of the complexity of the environment, are typically out of our control. And our wish is to invent models of system-environemt interactions capturing faithfully the key properties of the dynamics of the system alone. For a general discussion on the models of open system dynamics we refer to $6,6,8$, 8 . In what follows we will restrict our analysis to a particular toy model of the open system dynamics.

In the rest of this section we will introduce the model and elementary properties of quantum channels. In the following two sections we will discuss thermalization and decoherence processes within this model. In Section IV we will focus on entanglement created in the discussed collision models and finally, in the Section V we will derive master equations for these collision processes.

\subsection{Simple collision model}

Let $\mathcal{H}$ be the Hilbert space of the system of interest. States are identified with elements of the set of density operators (positive operators of unit trace)

$$
\mathcal{S}(\mathcal{H})=\{\varrho: \varrho \geq O, \operatorname{tr}[\varrho]=1\} .
$$

We will assume that environment consists of huge number of particles, each of them described by the same Hilbert space $\mathcal{H}$. In a sense, it forms a reservoir of particles. Moreover, we assume that initially they are all in the same state $\xi$, i.e. the initial state of the environment/reservoir is $\omega=\xi^{\otimes N}$. In each time step the system interacts with a single environment's particle. The interaction is described by a unitary operator $U$ acting on joint Hilbert space $\mathcal{H} \otimes \mathcal{H}$. Thus, the one-step system's evolution is described by a map

$$
\varrho \rightarrow \varrho^{\prime}=\mathcal{E}[\varrho]=\operatorname{tr}_{\mathrm{env}}\left[U(\varrho \otimes \xi) U^{\dagger}\right],
$$

where $\varrho$ is the initial state of the system and $\operatorname{tr}_{\text {env }}$ denotes the partial trace over the environment. In each time step a system undergoes a collision with a single particle from the reservoir. In a realistic scenarios these collisions are happening 


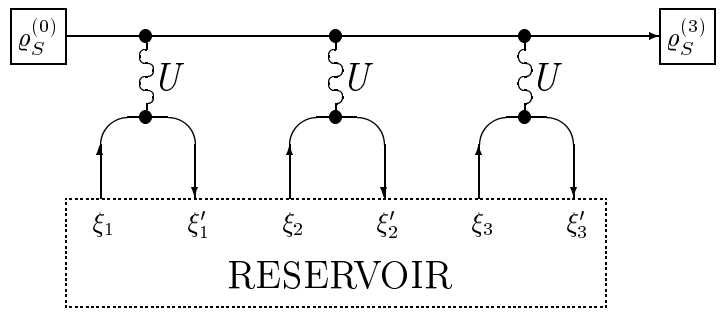

Figure 1: A simple collision model.

randomly, but we will assume that each reservoir's particle interacts with the system at most once (see Fig. 1). Thus, the system evolution is driven by a sequence of independent collisions. After the $n$th collision we get

$$
\varrho_{0} \rightarrow \varrho_{n}^{\prime}=\operatorname{tr}_{\mathrm{env}}\left[U\left(\varrho_{n-1} \otimes \xi\right) U^{\dagger}\right]=\mathcal{E}\left[\varrho_{n-1}\right]=\mathcal{E}^{n}\left[\varrho_{0}\right] .
$$

\subsection{Quantum channels}

The mapping $\mathcal{E}$ defined on the set of all operators $\mathcal{L}(\mathcal{H})$ satisfies the following properties:

1. $\mathcal{E}$ is linear, i.e. $\mathcal{E}[X+c Y]=\mathcal{E}[X]+c \mathcal{E}[Y]$ for all $X, Y \in \mathcal{L}(\mathcal{H})$ and all complex numbers $c$.

2. $\mathcal{E}$ is trace-preserving, i.e. $\operatorname{tr}[\mathcal{E}[X]]=\operatorname{tr}[X]$ for all operators $X$ with finite trace.

3. $\mathcal{E}$ is completely positive, i.e. $(\mathcal{E} \otimes \mathcal{I})[\Omega] \geq O$ for all positive operators $\Omega \in \mathcal{L}(\mathcal{H} \otimes \mathcal{H})$.

Due to Stinespring dilation theorem the inverse is also true. If a mapping $\mathcal{E}$ satisfies above three conditions, there is a unitary operator $U$ and a state $\xi$ such that the Eq. (3) holds. Such completely positive trace-preserving linear maps we call channels.

For any state $\xi \in \mathcal{S}(\mathcal{H})$ there exist a unit vector $\Psi \in \mathcal{H} \otimes \mathcal{H}$ such that $\xi=\operatorname{tr}_{2}|\Psi\rangle\langle\Psi|$, where $\operatorname{tr}_{2}$ is the partial trace over the second subsystem. We call $\Psi$ a purification of $\xi$. It is straightforward to verify that the following identity holds

$$
\mathcal{E}[\varrho]=\operatorname{tr}_{\mathrm{env}}\left[U(\varrho \otimes \xi) U^{\dagger}\right]=\operatorname{tr}_{\mathrm{env}^{\prime}}\left[(U \otimes I)(\varrho \otimes|\Psi\rangle\langle\Psi|)(U \otimes I)^{\dagger}\right] .
$$

Let $\varphi_{j}$ is an orthonormal basis on $\mathcal{H} \otimes \mathcal{H}$ and define linear operators $A_{j}=$ $\left\langle\varphi_{j} \mid(U \otimes I) \Psi\right\rangle$ acting on the system. Then the right-hand side of the above equation reads

$$
\mathcal{E}[\varrho]=\sum_{j}\left\langle\varphi_{j} \mid(U \otimes I) \Psi\right\rangle \varrho\left\langle(U \otimes I) \Psi \mid \varphi_{j}\right\rangle=\sum_{j} A_{j} \varrho A_{j}^{\dagger} .
$$


By definition the so-called Kraus operators $A_{j}$ satisfy the normalization $\sum_{j} A_{j}^{\dagger} A_{j}=$ I. Any mapping of the Kraus form $\mathcal{E}[\varrho]=\sum_{j} A_{j} \varrho A_{j}^{\dagger}$ for arbitrary set of operators $\left\{A_{1}, A_{2}, \ldots\right\}$ fulfilling the normalization constraint determines a valid quantum channel.

Channels with a single Kraus operator $\mathcal{E}[\varrho]=A \varrho A^{\dagger}$ are unitary channels, because the normalization $A^{\dagger} A=I$ implies that $A$ is unitary. Let us note that Kraus representation and also the number of Kraus operators are not unique. For more details we refer to [9, 10].

\section{Quantum homogenization as an analogue to thermalization}

The 0th law of thermodynamics postulates that an object brought in a contact with a reservoir of temperature $T$ will equalize its temperature with the reservoir's temperature. This process is known as the process of thermalization. Our attempt is to design a quantum analogue of this process.

The concept of temperature is the key ingredient of the thermalization process. In fact, as a consequence it is the temperature that describes a state of the system, that is, it contains the relevant part of the information needed for the investigation of the system's properties. What is the quantum analogue of the temperature? There are several ways how this concept can be introduced. One can use the connection between temperature and the entropy, or energy per single particle. By choosing such analogue, the thermalization can be defined as a process that leads a system with a given temperature $T_{S}$ to the temperature of the reservoir $T_{\xi}$. In classical theory this process is achieved because of mutual interactions/collisions between the particles. In the process of collision they mutually (partly) exchange their microscopic properties (energy, momentum). Subsequently, the temperature of the system being in a contact with the reservoir is changing and finally (approximately) equalize $T_{\xi}$. Let us note that we have used the temperature as a property that can be attributed even to a single particle, however, such definition is meaningful only in the statistical sense.

In quantum theory the state of a quantum system is described with a density operator. This operator contains all the information. Therefore, we choose single-particle density operator to play the role of the quantum temperature in our quantum analogue. So the goal is to design a model of the dynamics of the system+reservoir such that the initial state of the system particle $\varrho_{S}$ evolves into the state of the reservoir particles $\xi$. Because of the property that finally all particles are described by the same state $\xi$ we will refer to this process as to homogenization instead of the thermalization. In fact this notion is more appropriate to characterize the features of our model.

Ideally the homogenization should implement the following transformation

$$
\varrho_{S} \otimes \xi \otimes \cdots \otimes \xi \mapsto \xi_{S} \otimes \xi \otimes \cdots \otimes \xi,
$$

for all $\varrho$ and $\xi$. For finite reservoirs this process will perform $N \rightarrow N+1$ 
cloning transformation, which would violate the quantum no-cloning theorem [1, 12. Therefore, this process cannot be achieved in any dynamical model respecting the quantum theory. We will relax our requirements and investigate whether the homogenization can be implemented approximately via a sequence of (independent) collisions. Moreover, if initially $\varrho=\xi$, meaning there is nothing to homogenize, then the evolution is trivial. Let us summarize our assumptions and problem.

Definition 1. We call an interaction $U$ a generator of $\delta$-homogenization $\delta>0$ is the following conditions are satisfied

- Trivial homogenization. The joint state of the system and the environment after $n$th interaction reads

$$
\Omega_{n}=U_{n} \cdots U_{1}\left(\varrho_{S} \otimes \xi^{\otimes n}\right) U_{1}^{\dagger} \cdots U_{n}^{\dagger},
$$

where $U_{j}=U_{S, j} \otimes I_{\mathrm{env} \backslash\{j\}}$ describes the $j$ th interaction $\left(I_{\mathrm{env}} \backslash\{j\}\right.$ is the identity operator on the environment excluding the $j$ th particle). The for all $\xi \in \mathcal{S}(\mathcal{H})$

$$
\xi_{S} \otimes \xi \otimes \cdots \otimes \xi \mapsto \Omega_{n}=\xi_{S} \otimes \xi \otimes \cdots \otimes \xi .
$$

- Convergence. Let $\varrho_{S}^{(n)}$ be the state of the system after $n$th collision. Then there exist $N_{\delta}$ such that for all $n>N_{\delta}$

$$
D\left(\varrho_{S}^{(n)}, \xi\right) \leq \delta .
$$

- Stability of the reservoir. Let $\xi_{j}^{\prime}$ be the state of the $j$ th reservoir's particle after the its interaction with the system. Then

$$
D\left(\xi_{j}^{\prime}, \xi\right) \leq \delta,
$$

for all $j$.

Our task is simple: find $U$ satisfying the above properties.

\subsection{Trivial homogenization}

Let us denote by $\mathcal{H}_{ \pm}$the symmetric and antisymmetric subspaces of $\mathcal{H} \otimes \mathcal{H}$. A vector $\Phi$ belongs to $\mathcal{H}_{ \pm}$if $S \Phi= \pm \Phi$, where $S$ is the swap operator defined as $S=\sum_{j k}\left|\varphi_{j} \otimes \varphi_{k}\right\rangle\left\langle\varphi_{k} \otimes \varphi_{j}\right|$ for arbitrary orthonormal basis $\left\{\varphi_{j}\right\}$ of $\mathcal{H}$. It follows that for all $\varrho, \xi \in \mathcal{S}(\mathcal{H})$

$$
S(\varrho \otimes \xi) S^{\dagger}=\xi \otimes \varrho .
$$

Let us denote by $P_{ \pm}$projections onto symmetric and antisymmetric subspace. Then $S=P_{+}-P_{-}$. 
The condition of trivial homogenization implies that

$$
U|\psi \otimes \psi\rangle=|\psi \otimes \psi\rangle
$$

for all unit vectors $\psi \in \mathcal{H}$. This determines the action of the transformation $U$ on the symmetric subspace $\mathcal{H}_{+}$. However, it does not give any constraint on the antisymmetric subspace $\mathcal{H}_{-}$. Since $\mathcal{H} \otimes \mathcal{H}=\mathcal{H}_{+} \oplus \mathcal{H}_{-}$we can write $U=e^{i \gamma} I_{+} \oplus V_{-}$, where $V_{-}$is arbitrary unitary operator on the subspace $\mathcal{H}_{-}$and $I_{+}$is the identity operator on the symmetric subspace $\mathcal{H}_{+}$. In fact, $I_{+}=\left.P_{+}\right|_{\mathcal{H}_{+}}$. Any of the collisions of the form $U=e^{i \gamma} I_{+} \oplus V_{-}$fulfills the conditions of trivial homogenization.

Let us consider the case of $d=\operatorname{dim} \mathcal{H}=2$ (qubit), for which the antisymmetric space is one dimensional. In one-dimensional Hilbert spaces the unitary operators are complex square roots of the unity, i.e. $V_{-}=e^{i \beta} I_{-}$. Explicitly the transformation can be expressed as

$$
\begin{aligned}
U|00\rangle & =e^{i \gamma}|00\rangle, \\
U|11\rangle & =e^{i \gamma}|11\rangle, \\
U(|01\rangle+|10\rangle) & =e^{i \gamma}(|01\rangle+|10\rangle), \\
U(|01\rangle-|10\rangle) & =e^{i(\gamma+\beta)}(|01\rangle-|10\rangle) .
\end{aligned}
$$

Using the identities $e^{i \gamma}=e^{i(\gamma+\beta / 2)} e^{-i \beta / 2}, e^{i(\gamma+\beta)}=e^{i(\gamma+\beta / 2)} e^{i \beta / 2}$ and discarding the irrelevant global phase factor $e^{i(\gamma+\beta / 2)}$ we get a one-parametric set of solutions

$$
U_{\eta}=\cos \eta I+i \sin \eta S,
$$

where we set $\eta=-\beta / 2$.

In case of more dimensional space (where $d>2$ ) the set of unitary transformations is larger. However, when we fix $V_{+}=e^{i \beta} I_{-}$, we get the same oneparametric set of solutions

$$
U_{\eta}=\cos \eta I+i \sin \eta S=e^{i \eta S} .
$$

Any element of this class of unitary operators we will call a partial swap. In what follows we will focus on the collision dynamics generated by partial swap collisions.

\subsection{Partial swap collisions}

In what follows we denote $\sin \eta=s$ and $\cos \eta=c$. In the process of homogenization, the system particle interacts sequentially with one of the $N$ particles of the reservoir through the transformation $U_{\eta}=c I+i s S$. After the first collision the system and the first reservoir particle undergo the evolution

$$
\varrho_{S}^{(0)} \otimes \xi \mapsto U_{\eta}\left(\varrho_{S}^{(0)} \otimes \xi\right) U_{\eta}^{\dagger}=c^{2} \varrho_{S}^{(0)} \otimes \xi+s^{2} \xi \otimes \varrho_{S}^{(0)}+i c s\left[S, \varrho_{S}^{(0)} \otimes \xi\right] .
$$


The states of the system particle and of the reservoir particle are obtained as partial traces. Specifically, after the system particle is in the state described by the density operator

$$
\varrho_{S}^{(1)}=c^{2} \varrho_{S}^{(0)}+s^{2} \xi+i c s\left[\xi, \varrho_{S}^{(0)}\right],
$$

while the first reservoir particle is now in the state

$$
\xi_{1}^{\prime}=s^{2} \varrho_{S}^{(0)}+c^{2} \xi+i c s\left[\varrho_{S}^{(0)}, \xi\right] .
$$

When we recursively apply the partial-swap transformation then after the interaction with the $n$-th reservoir particle, we obtain

$$
\varrho_{S}^{(n)}=c^{2} \varrho_{S}^{(n-1)}+s^{2} \xi+i c s\left[\xi, \varrho_{S}^{(n-1)}\right],
$$

as the expression for the density operator of the system particle, while the $n$-th reservoir particle is in the state

$$
\xi_{n}^{\prime}=s^{2} \varrho_{S}^{(n-1)}+c^{2} \xi+i c s\left[\varrho_{S}^{(n-1)}, \xi\right] .
$$

\subsection{System's convergence}

Our next task is to analyze the remaining conditions on the homogenization process. First task is to show that $\varrho_{S}^{(n)}$ monotonically converges to a $\delta$-vicinity of $\xi$ for all parameters $\eta \neq 0$. We will utilize the Banach fixed point theorem [13] claiming that iterations of a strictly contractive mapping converge to the single-point contraction. This point is unique and determined by the condition $\mathcal{E}[\xi]=\xi$. A channel $\mathcal{E}$ is called strictly contractive if it fulfills the inequality $D(\mathcal{E}[\varrho], \mathcal{E}[\xi]) \leq k D(\varrho, \xi)$ with $0 \leq k<1$ for all $\varrho, \xi \in \mathcal{S}$.

First of all, it is easy to verify that $\xi$ is a fixed point of $\mathcal{E}_{\xi}$, where $\mathcal{E}_{\xi}$ is defined by the Eq.(16). As a distance we will use the Hilbert-Schmidt distance

$$
D\left(\varrho_{1}, \varrho_{2}\right)=\left\|\varrho_{1}-\varrho_{2}\right\|=\sqrt{\operatorname{tr}\left[\varrho_{1}^{2}\right]+\operatorname{tr}\left[\varrho_{2}^{2}\right]-2 \operatorname{tr}\left[\varrho_{1} \varrho_{2}\right]}
$$

where $\varrho_{1}, \varrho_{2}$ represent quantum states. Consequently,

$$
D^{2}\left(\mathcal{E}\left[\varrho_{1}\right], \mathcal{E}\left[\varrho_{2}\right]\right)=c^{4} D^{2}\left(\varrho_{1}, \varrho_{2}\right)+c^{2} s^{2}\left\|\left[\xi, \varrho_{1}-\varrho_{2}\right]\right\|^{2}
$$

where we used that $\mathcal{E}[\varrho]=c^{2} \varrho+s^{2} \xi+i c s[\xi, \varrho]$. Defining an operator $\Delta=\varrho_{1}-\varrho_{2}$ our aim is to prove the relation $\|[\xi, \Delta]\|^{2} \leq\|\Delta\|^{2}$. Assuming the spectral decomposition of $\xi$ equals $\xi=\sum_{k} \lambda_{k}|k\rangle\langle k|$ and performing the trace in the 
basis $|k\rangle$ we obtain

$$
\begin{aligned}
\|\xi \Delta-\Delta \xi\|^{2} & =\operatorname{tr}\left[(\xi \Delta-\Delta \xi)^{\dagger}(\xi \Delta-\Delta \xi)\right]=2 \operatorname{tr}\left[\xi^{2} \Delta^{2}\right]-2 \operatorname{tr}\left[(\xi \Delta)^{2}\right] \\
& =2 \sum_{j} \lambda_{j}^{2}\left\langle j\left|\Delta^{2}\right| j\right\rangle-2 \sum_{j, k} \lambda_{j} \lambda_{k}|\langle j|\Delta| k\rangle|^{2} \\
& =2 \sum_{j, k} \lambda_{j}^{2}\langle j|\Delta| k\rangle\langle k|\Delta| j\rangle-2 \sum_{j, k} \lambda_{j} \lambda_{k}|\langle j|\Delta| k\rangle|^{2} \\
& =\sum_{j, k}\left(2 \lambda_{j}^{2}-2 \lambda_{j} \lambda_{k}\right)|\langle j|\Delta| k\rangle|^{2} \\
& =\sum_{j, k}\left(\lambda_{j}^{2}+\lambda_{k}^{2}-2 \lambda_{j} \Lambda_{k}\right)|\langle j|\Delta| k\rangle|^{2}=\sum_{j, k}\left(\lambda_{j}-\lambda_{k}\right)^{2}|\langle j|\Delta| k\rangle|^{2} \\
& \leq \sum_{j, k}|\langle j|\Delta| k\rangle|^{2}=\operatorname{tr}\left[\Delta^{\dagger} \Delta\right]=\|\Delta\|^{2}
\end{aligned}
$$

where we employed the hermiticity of $\Delta\left(|\langle j|\Delta| k\rangle|^{2}=|\langle l|\Delta| j\rangle|^{2}\right)$ and positivity of $\xi$ which means $\left|\lambda_{1}-\lambda_{2}\right| \leq 1$. The proved inequality enables us to write

$$
D\left(\mathcal{E}\left[\varrho_{1}\right], \mathcal{E}\left[\varrho_{2}\right]\right) \leq|c| D\left(\varrho_{1}, \varrho_{2}\right)
$$

i.e. the contractivity coefficient $k$ is determined by the parameter $c=\cos \eta$ of the partial swap and the map $\mathcal{E}$ is contractive whenever $|c|<1$. This result is important because it ensures that in the limit of infinite steps the system will be not only $\delta$-homogenized, but will be described exactly by the state $\xi$ of the reservoir. It means the distance $D\left(\varrho_{S}^{(n)}, \xi\right) \rightarrow 0$ vanishes with the number of interactions $n$.

\subsection{Stability of the reservoir}

In order to satisfy the last condition (stability of the reservoir) we need to evaluate the distances $D\left(\xi_{j}^{\prime}, \xi\right)$. For each value of $\eta$ we can specify $\delta$ and $N_{\delta}$. However, the question is whether for arbitrary value of $\delta>0$ there is a suitable collision $U_{\eta}$ and how large is the value of $N_{\delta}$ for which $D\left(\varrho_{S}^{\left(N_{\delta}\right)}, \xi\right) \leq \delta$.

For the system we can combine the contractivity bound $D(\mathcal{E}[\varrho], \mathcal{E}[\xi]) \leq$ $|c| D(\varrho, \xi)$ with the fact that $\mathcal{E}[\xi]=\xi$ to obtain an estimate

$$
D\left(\xi, \varrho_{S}^{(n)}\right) \leq|c|^{n} D\left(\xi, \varrho_{S}^{(0)}\right)=\sqrt{2}|c|^{n} \leq \delta
$$

Consequently,

$$
N_{\delta}=\frac{\ln (\delta / \sqrt{2})}{\ln |c|},
$$

but the potential values of $\delta$ we need to specify from the stability of the reservoir. A direct calculation gives the following bound

$$
\begin{aligned}
D\left(\xi, \xi_{n}^{\prime}\right) & =\left\|\xi-c^{2} \xi-s^{2} \varrho_{S}^{(n-1)}-i c s\left[\varrho_{S}^{(n-1)}, \xi\right]\right\| \\
& \leq s^{2}|| \xi-\varrho_{S}^{(n-1)}\|+|c s| \cdot\|\left[\varrho_{S}^{(n-1)}, \xi\right] \| \\
& \leq s^{2} D\left(\xi, \varrho_{S}^{(n-1)}\right)+2|c s| \cdot\left\|\varrho_{S}^{(n-1)}\right\| \cdot\|\xi\| \\
& \leq \sqrt{2}|s c|\left(|s| \cdot|c|^{n-2}+\sqrt{2}\right) \\
& <\sqrt{2}|s c|(1+\sqrt{2}) \equiv \delta .
\end{aligned}
$$


Important is that $\delta$ can be arbitrarily small, hence $\delta$-homogenization is achievable for a restricted class of collisions $U_{\eta}$ satisfying the identity $(2+\sqrt{2})|\sin \eta \cos \eta| \leq$ $\delta$. The number of steps $N_{\delta}$ we achieve if the value of $\delta$ is inserted into the formula for $N_{\delta}$, i.e.

$$
N_{\delta} \approx \frac{\ln [(1+\sqrt{2})|s c|]}{\ln |c|} .
$$

Let us note that one can derive more precise expression for $N_{\delta}$ and $\delta$, but this is not important for our further purposes.

In summary, the class of partial swap operators $U_{\eta}$ satisfies the conditions for $\delta$-homogenization process.

Remark 1. Swap in the classical theory. Let us note that in the classical picture of thermalization process we also use model of mutual collisions of the system with its thermal reservoir. In these (inelastic) collisions the particles exchange energy and momentum. In some sense, this process can be understood as a transformation that partially "swaps" energy and momentum of involved particles. Thus, from this perspective the homogenizing properties of partial swap collisions are not that surprising and can be viewed as the quantum analogue of classical collisions.

\subsection{Invariance of single-particle average state}

In this section we will allow particles forming the reservoir interacts among each other, but interactions are always pairwise. Partial swap operators possess one unique property, which is important from the point of view of more realistic thermalization process and reservoir's stability. Consider $\Omega$ being an $n$-partite composite system. It can be written in the form

$$
\Omega=\varrho_{1} \otimes \cdots \otimes \varrho_{n}+\Gamma,
$$

where $\Gamma$ is a traceless hermitian operators and $\varrho_{j}=\operatorname{tr}_{\bar{j}} \Omega$ are the states of individual subsystems. Applying a partial swap collision between $j$ th and $k$ th subsystems we obtain a state

$$
\begin{aligned}
\Omega^{\prime} & =c^{2} \Omega+s^{2} S_{j k} \Omega S_{j k}+i c s\left[\Omega, S_{j k}\right] \\
& =\varrho_{1}^{\prime} \otimes \cdots \otimes \varrho_{n}^{\prime}+\Gamma^{\prime}
\end{aligned}
$$

where $S_{j k}$ denotes the swap operator between the subsystems $j$ and $k$,

$$
\begin{aligned}
\varrho_{j}^{\prime} & =c^{2} \varrho_{j}+s^{2} \varrho_{k}+i c s\left[\varrho_{j}, \varrho_{k}\right] \\
\varrho_{k}^{\prime} & =c^{2} \varrho_{k}+s^{2} \varrho_{j}-i c s\left[\varrho_{j}, \varrho_{k}\right] \\
\varrho_{l}^{\prime} & =\varrho_{l} \text { for } l \neq j, k,
\end{aligned}
$$

and $\Gamma^{\prime}$ is the traceless part. It is straightforward to see that

$$
\bar{\varrho}_{\text {one }}=\frac{1}{n} \sum_{j} \varrho_{j}=\frac{1}{n} \sum_{j} \varrho_{j}^{\prime}=\bar{\varrho}_{\text {one }}^{\prime}
$$


thus, in the collision model provided by partial swap collisions the average oneparticle state $\bar{\varrho}_{\text {one }}$ is preserved. If taken the single-particle state as the quantum analogue of temperature, then this property means that partial swap interactions preserves the "quantum" temperature.

\section{Quantum decoherence via collisions}

In this section we will focus on quantum dynamics of decoherence. Let us note that in literature on quantum information the decoherence is used to name any nonunitary channel and the channels we are interested in are called (generalized) phase-damping channels. In accordance with our previous work 44 we will understand by decoherence a channel with the following properties:

- Preservation of the diagonal elements of a density matrix with respect to a given (decoherence) basis. Let us denote by $\mathcal{B}=\left\{\varphi_{1}, \ldots, \varphi_{d}\right\}$ the decoherence basis of $\mathcal{H}$. Then

$$
\mathcal{E} \circ \operatorname{diag}_{\mathcal{B}}=\operatorname{diag}_{\mathcal{B}}
$$

where $\operatorname{diag}_{\mathcal{B}}$ is a channel diagonalizing density operators in the basis $\mathcal{B}$, i.e. $\varrho \rightarrow \operatorname{diag}_{\mathcal{B}}[\varrho]$.

- Vanishing of the off-diagonal elements of a density matrix with respect to a given (decoherence) basis. In particular, asymptotically the iterations of the decoherence channels results in the diagonalization of the density matrix, i.e.

$$
\lim _{n \rightarrow \infty} \mathcal{E}^{n}=\operatorname{diag}_{\mathcal{B}}
$$

Our tasks is similar as in the previous part: find collisions $U$ generating the decoherence in basis $\mathcal{B}$.

The basis preservation implies that

$$
\left|\varphi_{j} \otimes \varphi_{k}\right\rangle \mapsto e^{i \eta_{j k}}\left|\varphi_{j} \otimes \varphi_{j k}^{\prime}\right\rangle
$$

where $\eta_{j k}$ are phases and for each $j$ the unit vectors $\left\{\varphi_{j k}^{\prime}\right\}_{k}$ form an orthonormal basis of $\mathcal{H}$. It is not difficult to verify that the unitary operators describing the collisions have the following form

$$
U=\sum_{j}\left|\varphi_{j}\right\rangle\left\langle\varphi_{j}\right| \otimes V_{j}
$$

where $V_{j}=\sum_{k} e^{i \eta_{j k}}\left|\varphi_{j k}^{\prime}\right\rangle\left\langle\varphi_{k}\right|$ are unitary operators on individual systems of the environment. Such operators form a class of controlled unitaries with the system playing the role of the control system and the environment playing the role of the target system. 
A single controlled unitary collision induce the following channel on the system

$$
\mathcal{E}[\varrho]=\operatorname{tr}_{\mathrm{env}}\left[U(\varrho \otimes \xi) U^{\dagger}\right]=\sum_{j, k}\left|\varphi_{j}\right\rangle\left\langle\varphi_{j}|\varrho| \varphi_{k}\right\rangle\left\langle\varphi_{k}\right| \operatorname{tr}\left[V_{j} \xi V_{k}^{\dagger}\right] .
$$

Let us note an interesting feature that under the action of this channel the matrix elements of density operators do not mix, i.e. the output value of $\varrho_{j k}^{\prime}=$ $\left\langle\varphi_{j} \mid \mathcal{E}[\varrho] \varphi_{k}\right\rangle$ depends only on the value of $\varrho_{j k}=\left\langle\varphi_{j} \mid \varrho \varphi_{k}\right\rangle$. In order to fulfill the second decoherence condition it is sufficient to show that for $j \neq k$

$$
\left|\varrho_{j k}^{\prime}\right|<\left|\varrho_{j k}\right| .
$$

For any unitary operator $W=\sum_{l} e^{i w_{l}}|l\rangle\langle l|$

$$
|\operatorname{tr}[\xi W]|=\left|\sum_{l} e^{i w_{l}}\langle l|\xi| l\rangle\right|=\left|\sum_{l} p_{l} e^{i w_{l}}\right| \leq 1,
$$

where $p_{l}=\langle l|\xi| l\rangle$ are probabilities. This inequality is saturated only if $\xi$ is an eigenvector of $W$. It follows that the inequality

$$
\left|\varrho_{j k}^{\prime}\right|=\left|\varrho_{j k}\right| \cdot\left|\operatorname{tr}\left[V_{j} \xi V_{k}^{\dagger}\right]\right| \leq\left|\varrho_{j k}\right|
$$

holds, hence the off-diagonal elements are non-increasing. They are strictly decreasing if and only if $\left|\operatorname{tr}\left[V_{j} \xi V_{k}^{\dagger}\right]\right|<1$ for all $j \neq k$, which is the case if $\xi$ is not an eigenvector of any of the operators $V_{k}^{\dagger} V_{j}$.

In summary, we have shown that decoherence processes are intimately related with controlled unitary operators. In particular, the decoherence processes are generated by controlled unitary collisions $U=\sum_{j}\left|\varphi_{j}\right\rangle\left\langle\varphi_{j}\right| \otimes V_{j}$ satisfying $\left|\operatorname{tr}\left[V_{j} \xi V_{k}^{\dagger}\right]\right|<1$ for all $j \neq k$. In such case

$$
\lim _{n \rightarrow \infty} \mathcal{E}^{n}[\varrho]=\operatorname{diag}_{\mathcal{B}}[\varrho]
$$

where $\operatorname{diag}_{\mathcal{B}}$ is a channel diagonalizing the input density operator in the basis $\mathcal{B}$. Let us note that there are always states $\xi$ (eigenvectors of $V_{k}^{\dagger} V_{j}$ ) for which the decoherence is not achieved, because for them some of the off-diagonal elements are preserved. On the other side, for each controlled unitary collision $U$ there are always states of the environment $\xi$ inducing the decoherence of all off-diagonal matrix elements.

\subsection{Simultaneous decoherence of the system and the en- vironment}

Could it happen that both the environment and the system are decohering simultaneously? In the same decoherence basis? We will see that answers to both these questions are positive. 
For the considered collision model driven by controlled unitary interactions we obtain that the evolution of the particles in the reservoir is described by the following channels

$$
\mathcal{N}[\xi]=\operatorname{tr}_{\text {sys }}\left[U(\varrho \otimes \xi) U^{\dagger}\right]=\sum_{j} \varrho_{j j} V_{j} \xi V_{j}^{\dagger} .
$$

Such channels are known as random unitary channels.

It is known that random unitary channels can describe decoherence channels and it was a surprising result that decoherences are not necessarily random unitary channels [15]. We do not need to go into the details of this relation to see that both the system and the environment can decohere. It follows from our previous discussion that environment will decohere if $U$ is controlled unitary transformation of the form

$$
U=\sum_{j} U_{j} \otimes\left|\psi_{j}\right\rangle\left\langle\psi_{j}\right|,
$$

where $\left\{\psi_{j}\right\}$ is the decoherence basis of the particles in the environment. That is, the question is whether there are unitary operators that can be written as

$$
U=\sum_{j} U_{j} \otimes\left|\psi_{j}\right\rangle\left\langle\psi_{j}\left|=\sum_{j}\right| \varphi_{j}\right\rangle\left\langle\varphi_{j}\right| \otimes V_{j}
$$

for suitable decoherence bases $\left\{\varphi_{j}\right\},\left\{\psi_{j}\right\}$ and unitary operators $U_{j}, V_{j}$.

Example 1. CTRL-NOT.

$$
\begin{aligned}
U_{\text {ctrl-NOT }} & =|0\rangle\langle 0|\otimes I+| 1\rangle\left\langle 1\left|\otimes \sigma_{x}=I \otimes\right|+\right\rangle\left\langle+\left|+\sigma_{z} \otimes\right|-\right\rangle\langle-| \\
& =|0+\rangle\langle 0+|+| 0-\rangle\langle 0-|+| 1+\rangle\langle 1+|-| 1-\rangle\langle 1-|,
\end{aligned}
$$

where $| \pm\rangle=(|0\rangle \pm|1\rangle) / \sqrt{2}$.

Proposition 1. A unitary operator $U$ describes a collision with simultaneous decoherence of both interacting systems if and only if $U=\sum_{j}\left|\varphi_{j}\right\rangle\left\langle\varphi_{j}\right| \otimes V_{j}$ and the unitary operators $V_{j}$ commute with each other.

Proof. By definition, the simultaneous decoherence requires simultaneous preservation of decoherence bases $\left\{\varphi_{j}\right\}$ and $\left\{\psi_{k}\right\}$, i.e.

$$
\left|\varphi_{j} \otimes \psi_{k}\right\rangle \rightarrow e^{i \eta_{j k}}\left|\varphi_{j} \otimes \psi_{k}\right\rangle
$$

It follows that

$$
U=\sum_{j k} e^{i \eta_{j k}}\left|\varphi_{j}\right\rangle\left\langle\varphi_{j}|\otimes| \psi_{k}\right\rangle\left\langle\psi_{k}\right|
$$

and we can define

$$
U_{k}=\sum_{j} e^{i \eta_{j k}}\left|\varphi_{j}\right\rangle\left\langle\varphi_{j}\left|, \quad V_{j}=\sum_{k} e^{i \eta_{j k}}\right| \psi_{k}\right\rangle\left\langle\psi_{k}\right|,
$$


to get the required expression

$$
U=\sum_{k} U_{k} \otimes\left|\psi_{k}\right\rangle\left\langle\psi_{k}\left|=\sum_{j}\right| \varphi_{j}\right\rangle\left\langle\varphi_{j}\right| \otimes V_{j} .
$$

It is straightforward to see that operators $\left\{V_{j}\right\}$ and $\left\{U_{k}\right\}$ form sets of mutually commuting elements.

If the unitary operators $V_{j}$ commute with each other, then in their spectral form $V_{j}=\sum_{j} e^{i \eta_{j k}}\left|\psi_{k}\right\rangle\left\langle\psi_{k}\right|$. Clearly, $U=\sum_{j}\left|\varphi_{j}\right\rangle\left\langle\varphi_{j}\left|\otimes V_{j}=\sum_{k} U_{k} \otimes\right| \psi_{k}\right\rangle\left\langle\psi_{k}\right|$ with $U_{k}=\sum_{j} e^{i \eta_{j k}}\left|\varphi_{j}\right\rangle\left\langle\varphi_{j}\right|$. Therefore, $U$ generates decoherence in both interacting systems.

Setting $\psi_{k} \equiv \varphi_{k}$ the system's decoherence basis and the environment's decoherence basis coincide. In such case the system and the environment will decohere with respect to the same basis. An example of such interaction for the case of qubit is the CTRL-Z operator

$$
U_{\text {ctrl-Z }}=|0\rangle\langle 0|\otimes I+| 1\rangle\left\langle 1\left|\otimes \sigma_{z}=I \otimes\right| 0\right\rangle\left\langle 0\left|+\sigma_{z} \otimes\right| 1\right\rangle\langle 1|,
$$

generating decoherence in the computational basis $|0\rangle,|1\rangle$. Moreover, this collision is symmetric with respect to exchange of the role of the control and the target.

\section{Entanglement in collision models}

The phenomenon of quantum entanglement is considered as one of the key properties of quantum theory. Our aim is not to discuss the philosophical background of this concept [16, 17, but rather focus on the dynamics of entanglement in the considered collision models. We say a state is separable if it can be written as a convex combination of factorized states, i.e.

$$
\varrho_{\mathrm{sep}}=\sum_{j} p_{j} \xi_{1}^{(j)} \otimes \cdots \otimes \xi_{n}^{(j)} .
$$

A state is entangled if it is not separable. The question whether a given state is entangled, or separable turns out to be very difficult. Partial results are achieved for the system of qubits and therefore in this section we will restrict our discussion only to qubits. However, some of the qualitative properties will be extendible also to larger systems.

A tangle is a measure of bipartite entanglement defined as

$$
\tau(\omega)=\min _{\omega=\sum_{j} p_{j}\left|\psi_{j}\right\rangle\left\langle\psi_{j}\right|} \sum_{j} p_{j} \tau\left(\psi_{j}\right)
$$

where $\tau(\psi)=S_{\operatorname{lin}}\left(\operatorname{tr}_{1}|\psi\rangle\langle\psi|\right)=2\left(1-\operatorname{tr}\left[\left(\operatorname{tr}_{1}|\psi\rangle\langle\psi|\right)^{2}\right]\right)=4 \operatorname{det} \operatorname{tr}_{1}[|\psi\rangle\langle\psi|]$ is the linear entropy of the state of one of the subsystems. Let us note that for 
pure bipartite states $S_{\operatorname{lin}}\left(\operatorname{tr}_{1}|\psi\rangle\langle\psi|\right)=S_{\operatorname{lin}}\left(\operatorname{tr}_{2}|\psi\rangle\langle\psi|\right)$. Due to seminal paper of Wootters [18] $\tau(\omega)=C^{2}(\omega)$, where $C(\omega)$ is the so-called concurrence

$$
C=\max \left\{0,2 \max \left\{\sqrt{\lambda_{j}}\right\}-\sum_{j} \sqrt{\lambda_{j}}\right\},
$$

where $\left\{\lambda_{j}\right\}$ are the eigenvalues of $R=\omega\left(\sigma_{y} \otimes \sigma_{y}\right) \omega^{*}\left(\sigma_{y} \otimes \sigma_{y}\right)$.

For multiqubit systems the tangle satisfies the so-called monogamy relation (originally conjectures by Coffman et al.[19] and proved by Osborne et al. 20])

$$
\sum_{k, k \neq j} \tau_{j k} \leq \tau_{j}
$$

where $\tau_{j} \equiv \tau_{j \bar{j}}$ and $\bar{j}$ denotes the set of all qubits except the $j$ th one, i.e. $\tau_{j}$ is a tangle between the $j$ th qubit and rest of the qubits considered as a single system. The above inequality is called $C K W$ inequality. If the multiqubit system is in a pure state $\Psi$ then

$$
\tau_{j}=4 \operatorname{det} \operatorname{tr}_{\bar{j}}[|\Psi\rangle\langle\Psi|] .
$$

Example 2. Saturation of $C K W$ inequalities. At the end of the original paper [19] it is stated that states in the subspace covered by the basis $\left\{|1\rangle_{j} \otimes\left|0^{\otimes N-1}\right\rangle_{\bar{j}}\right\}$ saturates the CKW inequalities. In what follows we will show that any state of the form

$$
|\Psi\rangle=\alpha_{0}\left|0^{\otimes N}\right\rangle+\sum_{j=1}^{N} \alpha_{j}|1\rangle_{j} \otimes\left|0^{\otimes(N-1)}\right\rangle
$$

saturates the CKW inequalities.

The reduced bipartite density matrices are of the form

$$
\varrho=\left(\begin{array}{cccc}
a & d & e & 0 \\
d^{*} & b & f & 0 \\
e^{*} & f^{*} & c & 0 \\
0 & 0 & 0 & 0
\end{array}\right)
$$

and only one element determines the tangle of such state, namely

$$
\tau(\varrho)=4 f f^{*} .
$$

It follows that only the matrix element standing with the $|01\rangle\langle 10|$ term will be important for us. Direct calculations lead us to value $f=\alpha_{j} \alpha_{k}^{*}$ for the pair of $j$ th and $k$ th, hence

$$
\tau_{j k}=4\left|\alpha_{j}\right|^{2}\left|\alpha_{k}\right|^{2} .
$$

In the next step we evaluate the tangle between the $j$-th particle and the rest of the system. The state of single particle is described by matrix

$$
\varrho_{j}=\left(\begin{array}{cc}
\left|\alpha_{0}\right|^{2}+\sum_{k \neq j}\left|\alpha_{k}\right|^{2} & \alpha_{0} \alpha_{j}^{*} \\
\alpha_{j} \alpha_{0}^{*} & \left|\alpha_{j}\right|^{2}
\end{array}\right)
$$


Now it is easy to check that

$$
\tau_{j}=4 \operatorname{det} \varrho_{j}=\left|\alpha_{j}\right|^{2} \sum_{k \neq j}\left|\alpha_{k}\right|^{2}=\sum_{k \neq j} \tau_{j k}
$$

and therefore the CKW inequalities are saturated, i.e. $\Delta_{j}=\tau_{j}-\sum_{k \neq j} \tau_{j k}=0$ for all $j$.

\subsection{Entanglement in partial swap collision model}

A general pure input state equals

$$
|\Psi\rangle=|\psi\rangle_{S} \otimes|\varphi\rangle_{1} \otimes \cdots \otimes|\varphi\rangle_{N} .
$$

Since $[I, V \otimes V]=[S, V \otimes V]=0$ for all unitary operators $V$ on $\mathcal{H}$ it follows that also $\left[U_{\eta}, V \otimes V\right]=0$ for all unitary $V$. Moreover, the value of entanglement is invariant under local unitary transformations. Therefore, without loss of generality we can set $|\varphi\rangle=|0\rangle$ and $|\psi\rangle=\alpha|0\rangle+\beta|1\rangle$. After $n$ interactions

$\left|\Psi_{n}\right\rangle=\alpha\left|0^{\otimes(N+1)}\right\rangle+\beta c^{n}|1\rangle_{S} \otimes\left|0^{\otimes N}\right\rangle+\beta \sum_{l=1}^{n}|1\rangle_{l} \otimes\left|0^{\otimes N_{\bar{l}}}\right\rangle\left[i s c^{l-1} e^{i \eta(n-l)}\right]$,

where $N_{\bar{l}}$ denotes a system of $N$ qubits obtained by replacing the $l$ th qubit from the reservoir by the system qubit.

Since $\left|\Psi_{n}\right\rangle$ belongs to the class of states discussed in Example 2, we know that the state saturates $\mathrm{CKW}$ inequalities. We can used the derived formulas to write

$$
\begin{aligned}
\tau_{j k}(n) & =4|\beta|^{4} s^{4} c^{2(j+k-2)} ; \\
\tau_{0 k}(n) & =4|\beta|^{4} s^{2} c^{2(n+k-1)} \\
\tau_{j}(n) & =4|\beta|^{4} s^{2} c^{2(j-1)}\left(1-s^{2} c^{2(j-1)}\right) ; \\
\tau_{0}(n) & =4|\beta|^{4} c^{2 n}\left(1-c^{2 n}\right) .
\end{aligned}
$$

Let us note that these formulas are valid only if $j, k \leq n$. Otherwise the quantities vanish.

These results show that the system particle acts as a mediator entangling the reservoir particles which have never interacted directly. It is obvious that the later the two reservoir qubits interact with the system particle, the smaller the degree of their mutual entanglement is. Nevertheless, this value remains constant and does not depend on the subsequent evolution of the collision model (i.e., it does not depend on the number of interactions $n$ ). On the other hand the entanglement between the system particle and $k$ th reservoir particle $(k$ arbitrary) increases at the moment of interaction and then monotonously decreases with the number of interaction steps. 


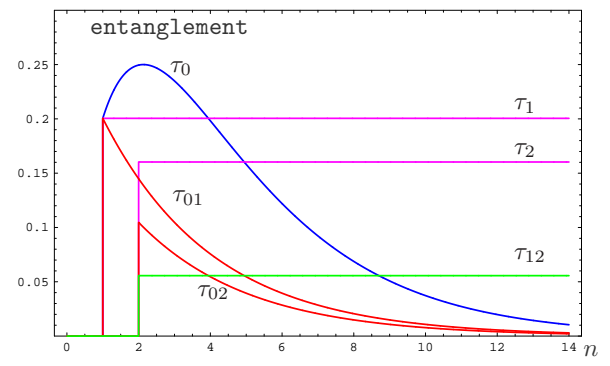

Figure 2: Entanglement in the homogenization process.

\subsection{Entanglement in controlled unitary collision model}

As before, let us assume that initially the system and the reservoir of $N$ qubits are described by a pure input state

$$
|\Omega\rangle=|\psi\rangle_{S} \otimes|\varphi\rangle_{1} \otimes \cdots \otimes|\varphi\rangle_{N},
$$

with $\psi=\alpha|0\rangle+\beta|1\rangle$. After $n$ controlled unitary collisions we get

$$
\left|\Omega_{n}\right\rangle=\left[\alpha|0\rangle \otimes\left|\varphi_{0}^{\otimes n}\right\rangle+\beta|1\rangle \otimes\left|\varphi_{1}^{\otimes n}\right\rangle\right] \otimes\left|\varphi^{\otimes(N-n)}\right\rangle,
$$

where we set $\left|\varphi_{0}\right\rangle=V_{0}|\varphi\rangle$ and $\left|\varphi_{1}\right\rangle=V_{1}|\varphi\rangle$.

Tracing out the system we end up with the reservoir described by the state

$$
\omega_{\text {env }}(n)=\left(|\alpha|^{2}\left|\varphi_{0}^{\otimes n}\right\rangle\left\langle\left.\varphi_{0}^{\otimes n}|+| \beta\right|^{2} \mid \varphi_{1}^{\otimes n}\right\rangle\left\langle\varphi_{1}^{\otimes n}\right|\right) \otimes|\varphi\rangle\left\langle\left.\varphi\right|^{\otimes(N-n)},\right.
$$

which is clearly separable, i.e. $\tau_{j k}(n)=0$. In this case no entanglement is created within the environment.

A state of the system qubit and $k$ th qubit from the reservoir equals (providing that $n \geq k$ )

$$
\begin{aligned}
\varrho_{0 k}(n)= & |\alpha|^{2}\left|0 \varphi_{0}\right\rangle\left\langle\left. 0 \varphi_{0}|+| \beta\right|^{2} \mid 1 \varphi_{1}\right\rangle\left\langle 1 \varphi_{1}\right| \\
& +\alpha \beta^{*}\left|\left\langle\varphi_{0} \mid \varphi_{1}\right\rangle\right|^{(n-1)}\left|0 \varphi_{0}\right\rangle\left\langle 1 \varphi_{1}\right|+c . c,
\end{aligned}
$$

thus for the tangle we have

$$
\tau_{0 k}(n)=4|\alpha|^{2}|\beta|^{2}\left|\left\langle\varphi_{0} \mid \varphi_{1}\right\rangle\right|^{2(n-1)}\left|\left\langle\varphi_{0} \mid \varphi_{1}^{\perp}\right\rangle\right|^{2} .
$$

If $n<k$ then $\tau_{0 k}(n)=0$. Further,

$$
\begin{aligned}
& \varrho_{0}(n)=|\alpha|^{2}|0\rangle\left\langle\left. 0|+| \beta\right|^{2} \mid 1\right\rangle\left\langle\left. 1\left|+\alpha \beta^{*}\right|\left\langle\varphi_{0} \mid \varphi_{1}\right\rangle\right|^{n} \mid 0\right\rangle\langle 1|+\text { c.c. }, \\
& \varrho_{k}(n)=|\alpha|^{2}\left|\varphi_{0}\right\rangle\left\langle\left.\varphi_{0}|+| \beta\right|^{2} \mid \varphi_{1}\right\rangle\left\langle\varphi_{1}\right|,
\end{aligned}
$$

resulting in the following values for tangle

$$
\begin{aligned}
& \tau_{0}(n)=4|\alpha|^{2}|\beta|^{2}\left(1-\left|\left\langle\varphi_{0} \mid \varphi_{1}\right\rangle\right|^{2 n}\right), \\
& \tau_{k}(n)=4|\alpha|^{2}|\beta|^{2}\left|\left\langle\varphi_{0} \mid \varphi_{1}^{\perp}\right\rangle\right|^{2} .
\end{aligned}
$$




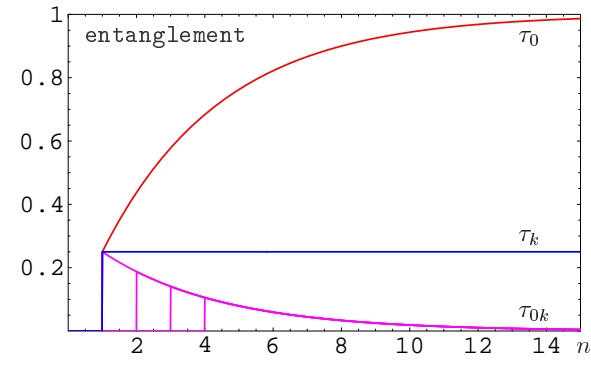

Figure 3: Entanglement in the decoherence process.

In summary, during the decoherence collision model the qubits in the reservoir are not entangled at all. Interaction of the system qubit with an individual reservoir's qubit entangles these pair of qubits. However, with the number of collision this bipartite entanglement vanishes and finally $\tau_{0 k}=0$ for all reservoir's qubits $k$. The entanglement between a fixed qubit of the reservoir and rest of the qubits preserves its value it gathered after the interaction. The entanglement between a system qubit and the reservoir is increasing with the number of collisions and approaches the maximal value $\tau_{0}(\infty)=4|\alpha|^{2}|\beta|^{2}$.

\section{Master equations for collision models}

Within the collision model the system undergoes a discrete dynamics

$$
\varrho \rightarrow \mathcal{E}_{1}[\varrho] \rightarrow \mathcal{E}_{2}[\varrho] \rightarrow \cdots \rightarrow \mathcal{E}_{n}[\varrho] \rightarrow \cdots,
$$

where $\mathcal{E}_{k}=\mathcal{E} \circ \cdots \circ \mathcal{E}=\mathcal{E}^{k}$. Let us define $\mathcal{E}_{0}$ as the identity map $\mathcal{I}$. It is straightforward to see that the sequence of channels $\mathcal{E}_{0}, \mathcal{E}_{1}, \mathcal{E}_{2}, \ldots$ form a discrete semigroup satisfying the relation

$$
\mathcal{E}_{n} \circ \mathcal{E}_{m}=\mathcal{E}_{n+m}
$$

for all $n, m=0,1,2, \ldots$. In this section we will investigate the continuous interpolations of such discrete semigroups. Interpreting the continuous parameter as time we will derive first order differential master equation generating the continuous sequence of linear maps. Our derivation of the master equation will be purely heuristic. We will simply replace $n$ in $\mathcal{E}_{n}$ by a continuous time parameter $t$ and after that we will have a look what are the consequences.

Let $\varrho_{t}=\mathcal{E}_{t}\left[\varrho_{0}\right]$. The time derivative of the state dynamics results in the following differential equation

$$
\frac{d \varrho_{t}}{d t}=\left(\frac{d \mathcal{E}_{t}}{d t}\right)\left[\varrho_{0}\right]=\left(\frac{d \mathcal{E}_{t}}{d t} \circ \mathcal{E}_{t}^{-1}\right)\left[\varrho_{t}\right]=\mathcal{G}_{t}\left[\varrho_{t}\right],
$$

where $\mathcal{G}_{t}$ is the generator of the dynamics $\mathcal{E}_{t}$. Let us note that this approach is very formal. There is no guarantee that the mapping $\mathcal{E}_{t}$ is a valid quantum 
channel for each value of $t$ and that the set $\mathcal{E}_{t}$ is indeed continuous. Another problematic issue is the assumed existence of the inverse of the map $\mathcal{E}_{t}^{-1}$ for all $t \geq 0$. All these properties need to be checked before we give some credit to the derived master equation.

\subsection{One-parametric semigroups}

In a special case when $\mathcal{G}_{t}$ is time-independent the solution of the differential equation

$$
\frac{d \varrho_{t}}{d t}=\mathcal{G}\left[\varrho_{t}\right]
$$

with the initial condition $\varrho_{t=0}=\varrho_{0}$ can be written in the form

$$
\varrho_{t}=e^{\mathcal{G} t}\left[\varrho_{0}\right] ; \quad \mathcal{E}_{t}=e^{\mathcal{G} t} .
$$

Since $e^{\mathcal{G}(t+s)}=e^{\mathcal{G} s} e^{\mathcal{G} t}$ it follows that

$$
\mathcal{E}_{t} \circ \mathcal{E}_{s}=\mathcal{E}_{t+s}
$$

for all $t, s \geq 0$ and $\mathcal{E}_{0}=\mathcal{I}$. In such case the continuous set of linear maps $\left\{\mathcal{E}_{t}\right\}$ form a one-parametric semigroup, which are playing important role in the theory of open system dynamics. Semigroups of channels are often used to approximate real open system's evolutions. In particular, they are satisfying the so-called Markovianity condition 21] restricting the memory effects of the environment. The following theorem specifies the properties of the generator $\mathcal{G}$ assuring the validity of the quantum channel constraints on $\mathcal{E}_{t}=e^{t \mathcal{G}}$.

Theorem 1. (Lindblad[22]) $\mathcal{E}_{t}=e^{\mathcal{G} t}$ are valid quantum channels for all $t \geq 0$ if and only if $\mathcal{G}$ can be written in the form

$$
\mathcal{G}[\varrho]=-\frac{i}{\hbar}[H, \varrho]+\frac{1}{2} \sum_{j k} c_{j k}\left(\left[\Lambda_{j} \varrho, \Lambda_{k}\right]+\left[\Lambda_{j}, \varrho \Lambda_{k}\right]\right),
$$

where $\left\{I, \Lambda_{1}, \ldots, \Lambda_{d^{2}-1}\right\}$ is an orthonormal operator basis consisting of hermitian traceless operators $\Lambda_{j}$, i.e. $\operatorname{tr}\left[\Lambda_{j} \Lambda_{k}\right]=\delta_{j k}$, the coefficients $c_{j k}$ form a positive matrix and $H$ is a hermitian traceless operator on $\mathcal{H}$.

\subsection{Divisibility of channels}

Any collision $U$ induced some channel $\mathcal{E}$. Our interpolation should smoothly connect this channel with the identity map $\mathcal{I}$ such that the connecting line is inside the set of channels. Since the set of channels is convex any two points are connected by a line containing only proper quantum channels, i.e. interpolation is surely possible although its continuity is still not guaranteed. Let us have a look on the possibility to interpolate $\mathcal{I}$ and $\mathcal{E}$ such that $\mathcal{E}=e^{\tau \mathcal{G}}$ for some time $\tau$ and time-independent Lindblad generator. Without loss of generality we can 
set $\tau=1$, i.e. $\mathcal{E}=e^{\mathcal{G}}$. The semigroup property implies that $\mathcal{E}=\mathcal{E}_{\varepsilon} \circ \mathcal{E}_{1-\varepsilon}$ for any $0<\epsilon<1$, or even stronger $\mathcal{E}=\mathcal{E}_{\epsilon}^{1 / \epsilon}$.

It is a surprising fact 23 that there are channels $\mathcal{E}$, which cannot be expressed as a nontrivial composition of some other channels $\mathcal{E}_{1}, \mathcal{E}_{2}$. Consequently not all channels can be part of some one-parametric semigroup. Before we will show one example, let us formally define the indivisibility.

Definition 2. We say a channel $\mathcal{E}$ is indivisible if $\mathcal{E}=\mathcal{E}_{1} \circ \mathcal{E}_{2}$ implies that either $\mathcal{E}_{1}$, or $\mathcal{E}_{2}$ are unitary channels (such decomposition is called trivial).

Let us note that one of seemingly counter-intuitive consequences is that unitary channels are indivisible. In a suitable representation the composition of channels is just a product of matrices. Thus for determinants

$$
\operatorname{det}\left(\mathcal{E}_{1} \circ \mathcal{E}_{2}\right)=\left(\operatorname{det} \mathcal{E}_{1}\right)\left(\operatorname{det} \mathcal{E}_{2}\right) .
$$

Let us denote by $\mathcal{E}_{\min }$ a channel minimizing the value of determinant, i.e. $\mathcal{E}_{\min }=$ $\arg \min _{\mathcal{E}} \operatorname{det} \mathcal{E}$. Assume $\mathcal{E}_{\text {min }}=\mathcal{E}_{1} \circ \mathcal{E}_{2}$. Since the value of $\operatorname{det} \mathcal{E}_{\text {min }}$ is negative and for channels $|\operatorname{det} \mathcal{E}| \leq 1$, it follows that either $\operatorname{det} \mathcal{E}_{1}=1$, $\operatorname{det} \mathcal{E}_{2}=\operatorname{det} \mathcal{E}_{\text {min }}$, or $\operatorname{det} \mathcal{E}_{2}=1, \operatorname{det} \mathcal{E}_{1}=\operatorname{det} \mathcal{E}_{\text {min }}$. But $\operatorname{det} \mathcal{E}=1$ implies $\mathcal{E}$ is a unitary channel, which proves the triviality of any decomposition of the minimum determinant channel. If $d=2$ (qubit), then one of the minimum determinant channels is the optimal universal NOT[23, i.e.

$$
\mathcal{E}_{\min }[\varrho]=\frac{1}{3}\left(I+\varrho^{T}\right),
$$

for which $\operatorname{det} \mathcal{E}_{\min }=-1 / 27$. The mapping $\varrho \rightarrow \varrho^{T}$ known as the universal NOT is not a proper quantum channel, because it is not completely positive and, thus, unphysical. Let us note that channels of minimal determinant are not the only indivisible channels. As far as the authors know a complete characterization of indivisible channels is not known.

For our purposes the introduced concept of indivisibility is not sufficient as we are interested in the existence of continuous semigroup between $\mathcal{I}$ and $\mathcal{E}$ generated by a unitary collision $U$. We need $\mathcal{E}$ to be infinitely divisible, i.e. $\mathcal{E}=\mathcal{E}_{1 / n}^{n}$ for all $n>0$. It was shown by Denisov 24] that infinitely divisible channel can be expressed in the form $\mathcal{E}=\mathcal{F} \circ e^{\mathcal{G}}$, where $\mathcal{F}$ is an idempotent channel $\left(\mathcal{F}^{2}=\mathcal{F}\right)$ satisfying $\mathcal{F G}=\mathcal{F} \mathcal{G} \mathcal{F}$ and $\mathcal{G}$ is a Lindblad generator. We are interested in cases when $\mathcal{F}=\mathcal{I}$, thus, $\mathcal{E}=e^{\mathcal{G}}$. Then it follows that the sequence of channels $\mathcal{E}^{n}=e^{n \mathcal{G}}$ determined by the collision model belongs to the oneparametric semigroup $e^{t \mathcal{G}}$. In other words a discrete semigroup is interpolated by a continuous one. We will see that this is the case for the collisions considered in the homogenization and decoherence processes.

For a given channel induced by a single collision a question of interest is whether $\mathcal{E}=e^{\mathcal{G}}$ for some Lindblad generator $\mathcal{G}$. In other words whether $\mathcal{G}=$ $\log \mathcal{E}$ is a valid generator of complete positive dynamics. Since channels $\mathcal{E}$ are not necessarily diagonalizable, the notion of the matrix/operator logarithm is 
not completely trivial. The logarithm is not unique and any operator $X$ such that $e^{X}=\mathcal{E}$ is called $\log \mathcal{E}$. We need to search all logarithms in order to find a valid generator of the Lindblad form. Evaluating the logarithm will give us a time-independent generator (if it exists) interpolating the discrete semigroup $\mathcal{E}_{n}=\mathcal{E}^{n}$. For both considered collision models the evaluation of the logarithm is not difficult. However, for illustrative purposes in our analysis of homogenization we will follow the ad hoc procedure exploiting (81), which (if successful) could capture also time-dependent generators. For the case of decoherence we will evaluate the logarithm of the induced channel.

\subsection{Bloch sphere parametrization}

The set of operators form a complex linear space endowed with a Hilbert-Schmidt scalar product $(X, Y)=\operatorname{tr}\left[X^{\dagger} Y\right]$. Let $\varphi_{1}, \ldots, \varphi_{d}$ be an orthonormal basis of $\mathcal{H}$. Then the set of $d^{2}$ operators $\left\{E_{j k}=\left|\varphi_{j}\right\rangle\left\langle\varphi_{k}\right|\right\}$ form an orthonormal operator basis, i.e. $\operatorname{tr}\left[E_{j^{\prime} k^{\prime}}^{\dagger} E_{j k}\right]=\left\langle\varphi_{j^{\prime}} \mid \varphi_{j}\right\rangle\left\langle\varphi_{k} \mid \varphi_{k^{\prime}}\right\rangle=\delta_{j j^{\prime}} \delta_{k k^{\prime}}$.

In what follows we will restrict to the case of two-dimensional Hilbert spaces $\mathcal{H}$ (qubits). This will be perfectly sufficient for our later purposes, because we will focus on qubit collision models of homogenization and decoherence. However, many of the properties and procedures can be easily extended to more dimensional case.

Let us start with a very convenient operator basis of qubit systems - the set of Pauli operators

$$
\begin{gathered}
I=|0\rangle\langle 0|+| 1\rangle\left\langle 1\left|, \quad \sigma_{x}=\right| 0\right\rangle\langle 1|+| 1\rangle\langle 0|, \\
\sigma_{y}=-i(|0\rangle\langle 1|-| 1\rangle\langle 0|), \quad \sigma_{z}=|0\rangle\langle 0|-| 1\rangle\langle 1|,
\end{gathered}
$$

where $\{|0\rangle,|1\rangle\}$ is an orthonormal basis of $\mathcal{H}$. These operators are hermitian, $\sigma_{j}=\sigma_{j}^{\dagger}$, and mutually orthogonal, i.e. $\operatorname{tr}\left[\sigma_{j} \sigma_{k}\right]=2 \delta_{j k}$ (we set $\sigma_{0}=I$ ). They are also unitary, but this property will not be very important for us.

Using Pauli operators the density operators takes the form

$$
\varrho=\frac{1}{2}(I+\vec{r} \cdot \vec{\sigma}),
$$

where $\vec{\sigma}=\left(\sigma_{x}, \sigma_{y}, \sigma_{z}\right)$ and $\vec{r}=\operatorname{tr}[\varrho \vec{\sigma}]$ is the so-called Bloch vector. The positivity constraint restricts its length $|\vec{r}| \leq 1$, i.e. in the Bloch vector parametrization the qubit's states form a unit sphere called Bloch sphere. Let us note that such simple picture of state space does not hold for more dimensional quantum systems.

A quantum channel $\mathcal{E}$ is acting on Pauli operators as follows

$$
\varrho \rightarrow \varrho^{\prime}=\mathcal{E}[\varrho]=\frac{1}{2}\left(\mathcal{E}[I]+x \mathcal{E}\left[\sigma_{x}\right]+y \mathcal{E}\left[\sigma_{y}\right]+z \mathcal{E}\left[\sigma_{z}\right]\right) .
$$

Using $\mathcal{E}\left[\sigma_{j}\right]=\sum_{k} \mathcal{E}_{k j} \sigma_{k}$ with $\mathcal{E}_{k j}=\frac{1}{2} \operatorname{tr}\left[\sigma_{k} \mathcal{E}\left[\sigma_{j}\right]\right]$. The tracepreservity ensures 
that $\mathcal{E}_{00}=\frac{1}{2} \operatorname{tr}[\mathcal{E}[I]]=1$ and $\mathcal{E}_{0 j}=\operatorname{tr}\left[\mathcal{E}\left[\sigma_{j}\right]\right]=0$ for $j=x, y, z$. That is,

$$
\varrho^{\prime}=\frac{1}{2}\left[I+\sum_{k=x, y, x}\left(\mathcal{E}_{k 0}+\sum_{j=x, y, x} \mathcal{E}_{k j} r_{j}\right) \sigma_{k}\right] .
$$

Comparing with the expression $\varrho^{\prime}=\frac{1}{2}\left(I+\vec{r}^{\prime} \cdot \vec{\sigma}\right)$ we get that under the action of a channel $\mathcal{E}$ the Bloch vectors are transformed by an affine transformation

$$
\vec{r} \rightarrow \vec{r}^{\prime}=\vec{t}+T \vec{r},
$$

where $t_{j}=\mathcal{E}_{j 0}$ and $T$ is a $3 \times 3$ matrix with entries $T_{j k}=\mathcal{E}_{j k}$ for $j, k=x, y, z$.

The Lindblad generator takes the form of $4 \times 4$ matrix

$$
\mathcal{G}=\left(\begin{array}{cccc}
0 & 0 & 0 & 0 \\
g_{10} & g_{11} & g_{12} & g_{13} \\
g_{20} & g_{21} & g_{22} & g_{23} \\
g_{30} & g_{31} & g_{32} & g_{33}
\end{array}\right),
$$

where $g_{j k}=\frac{1}{2} \operatorname{tr}\left[\sigma_{j} \mathcal{G}\left[\sigma_{k}\right]\right]$. Inserting the Lindblad operator-sum form

$$
\mathcal{G}[X]=-\frac{i}{\hbar} \sum_{j=x, y, z} h_{j}\left[\sigma_{j}, X\right]+\frac{1}{2} \sum_{j, k=x, y, z} c_{j k}\left(\left[\sigma_{j}, X \sigma_{k}\right]+\left[\sigma_{j} X, \sigma_{k}\right]\right)
$$

into the matrix expression we obtain

$$
\begin{aligned}
g_{j k} & =2 \sum_{l} \epsilon_{j k l} h_{l}+\frac{1}{2}\left(c_{k j}+c_{j k}\right)-\sum_{l} c_{l l} \delta_{j k}, \\
g_{k 0} & =i \sum_{j l} \epsilon_{j l k} c_{j l} .
\end{aligned}
$$

The inverse relations express the parameters $c_{j k}$ and $h_{j}$ via the elements of the matrix $\mathcal{G}$

$$
\begin{gathered}
h_{1}=\frac{g_{32}-g_{23}}{4}, \quad h_{2}=\frac{g_{13}-g_{31}}{4}, \quad h_{3}=\frac{g_{21}-g_{12}}{4} \\
c_{j j}=g_{j j}-\frac{1}{2} \sum_{k} g_{k k} \\
c_{12}=\frac{1}{2}\left(g_{12}+g_{21}-i g_{30}\right), \quad c_{21}=\frac{1}{2}\left(g_{12}+g_{21}+i g_{30}\right), \\
c_{23}=\frac{1}{2}\left(g_{23}+g_{32}-i g_{10}\right), \quad c_{32}=\frac{1}{2}\left(g_{23}+g_{32}+i g_{10}\right), \\
c_{13}=\frac{1}{2}\left(g_{13}+g_{31}+i g_{20}\right), \quad c_{31}=\frac{1}{2}\left(g_{13}+g_{31}-i g_{20}\right) .
\end{gathered}
$$

\subsection{Master equation for homogenization collision model}

The collision model of quantum homogenization driven by partial swaps results in the sequence of channels being powers of a channel $\mathcal{E}$ defined in Eq.(16), i.e.

$$
\vec{r} \rightarrow \vec{r}^{\prime}=c^{2} \vec{r}+s^{2} \vec{w}-c s \vec{w} \times \vec{r},
$$


where $\vec{w}$ is the Bloch vector associated with the state $\xi$ and $c=\cos \eta, s=\sin \eta$. Choosing a new operator basis $S_{j}=V \sigma_{j} V^{\dagger}$ such that $\xi=\frac{1}{2}\left(I+w S_{3}\right)$ we get

$$
\mathcal{E}=\left(\begin{array}{cccc}
1 & 0 & 0 & 0 \\
0 & c^{2} & c s w & 0 \\
0 & -c s w & c^{2} & 0 \\
s^{2} w & 0 & 0 & c^{2}
\end{array}\right)
$$

Let us note that the basis transformation $\sigma_{j} \rightarrow S_{j}$ corresponds to a rotation of the coordinate system of the Bloch sphere representation. Moreover, we used that the partial swap commutes with unitaries of the form $V \otimes V$, hence the form of the induced map (97) is unaffected, only the vectors are expressed with respect to different coordinate system.

Let us introduce an angle $\theta=\arctan (w s / c)=\arctan (w \tan \eta)$ and a parameter $q=\sqrt{c^{2}+w^{2} s^{2}}$. Using the identity $\cos \arctan (x)=1 / \sqrt{1+x^{2}}$ we get $q \cos \theta=\cos \eta=c$ and $q \sin \theta=w \sin \eta$, thus

$$
\mathcal{E}=\left(\begin{array}{cccc}
1 & 0 & 0 & 0 \\
0 & c q \cos \theta & c q \sin \theta & 0 \\
0 & -c q \sin \theta & c q \cos \theta & 0 \\
s^{2} w & 0 & 0 & c^{2}
\end{array}\right)
$$

The reason for such parametrization becomes clear if we evaluate powers of $\mathcal{E}$

$$
\mathcal{E}^{n}=\left(\begin{array}{cccc}
1 & 0 & 0 & 0 \\
0 & c^{n} q^{n} \cos n \theta & c^{n} q^{n} \sin n \theta & 0 \\
0 & -c^{n} q^{n} \sin n \theta & c^{n} q^{n} \cos n \theta & 0 \\
w\left(1-c^{2 n}\right) & 0 & 0 & c^{2 n}
\end{array}\right)
$$

In the next step we make ad hoc assumption and replace $n$ by $t / \tau$, where $\tau$ is some time scale and $t \geq 0$ is a continuous time parameter. Introducing the parameters

$$
\Omega=\theta / \tau, \quad c^{2 t / \tau}=e^{-\Gamma_{1} t}, \quad(c q)^{t / \tau}=e^{-\Gamma_{2} t},
$$

we end up with the continuous set of trace-preserving linear maps

$$
\mathcal{E}_{t}=\left(\begin{array}{cccc}
1 & 0 & 0 & 0 \\
0 & e^{-\Gamma_{2} t} \cos \Omega t & e^{-\Gamma_{2} t} \sin \Omega t & 0 \\
0 & -e^{-\Gamma_{2} t} \sin \Omega t & e^{-\Gamma_{2} t} \cos \Omega t & 0 \\
w\left(1-e^{-\Gamma_{1} t}\right) & 0 & 0 & e^{-\Gamma_{1} t}
\end{array}\right) .
$$

It is not difficult to see that they form a one-parametric semigroup $\left(\mathcal{E}_{t} \circ \mathcal{E}_{s}=\right.$ $\left.\mathcal{E}_{t+s}\right)$, but one needs to verify whether for each $t$ these maps are completely positive. Therefore, we calculate the generator $\mathcal{G}$ and verify its properties. In particular, we obtain a time-independent generator

$$
\mathcal{G}=\left(\begin{array}{cccc}
0 & 0 & 0 & 0 \\
0 & -\Gamma_{2} & -\Omega & 0 \\
0 & \Omega & -\Gamma_{2} & 0 \\
w \Gamma_{1} & 0 & 0 & -\Gamma_{1}
\end{array}\right)
$$


Using the Eqs.(96) we can verify that the parameters $h_{1}=h_{2}=0, h_{3}=-\Omega / 2$ are real and that the matrix

$$
C=\left(\begin{array}{ccc}
-\Gamma_{1} / 2 & -i 2 w \Gamma_{1} & 0 \\
i 2 w \Gamma_{1} & -\Gamma_{1} / 2 & 0 \\
0 & 0 & -\left(\Gamma_{1}+2 \Gamma_{2}\right) / 2
\end{array}\right)
$$

is positive, hence, the generator $\mathcal{G}$ is of Lindblad form. In the operator-sum (Kraus) form the master equation reads

$$
\begin{aligned}
\frac{d \varrho}{d t}= & i \frac{\Omega}{2 \hbar}\left[S_{z}, \varrho\right]-i w \Gamma_{1}\left(\Phi_{x y}[\varrho]+\Phi_{y x}[\varrho]\right) \\
& -\frac{\Gamma_{1}}{4}\left(\Phi_{x x}[\varrho]+\Phi_{y y}[\varrho]\right)-\frac{\Gamma_{1}+2 \Gamma_{2}}{4} \Phi_{x x}[\varrho]
\end{aligned}
$$

where $\Phi_{j k}[\varrho]=\frac{1}{2}\left(\left[\sigma_{j} \varrho, \sigma_{k}\right]+\left[\sigma_{j}, \varrho \sigma_{k}\right]\right)$. After a little algebra we obtain

$$
\begin{aligned}
\frac{d \varrho}{d t}= & i \frac{\Omega}{2 \hbar}\left[S_{z}, \varrho\right]-i w \Gamma_{1}\left(S_{x} \varrho S_{y}-S_{y} \varrho S_{x}+i \varrho S_{z}+i S_{z} \varrho\right) \\
& +\frac{1}{4} \Gamma_{1}\left(S_{x} \varrho S_{x}+S_{y} \varrho S_{y}-2 \varrho\right)+\frac{1}{4}\left(2 \Gamma_{2}-\Gamma_{1}\right)\left(S_{z} \varrho S_{z}-\varrho\right) .
\end{aligned}
$$

Let us note that for the special choice of parameters the above master equation coincide with the master equation used to model the spontaneous decay of a two-level atom. In particular, setting $\Gamma_{1}=2 \Gamma_{2}=2 \gamma=-\frac{2}{\tau} \ln \cos \eta$ and $\xi$ being a pure state $(w=1)$ we get

$$
\frac{d}{d t} \varrho=-i \frac{\Omega}{2 \hbar}\left[S_{z}, \varrho\right]+\frac{\gamma}{2}\left[2 S_{-} \varrho S_{+}-S_{-} S_{+} \varrho-\varrho S_{+} S_{-}\right)
$$

where we used $S_{ \pm}=\left(S_{x} \pm i S_{y}\right) / 2$.

\subsection{Master equation for decoherence collision model}

In this section we will repeat the same steps as in the previous one, but for collisions described by controlled unitary interactions. Without loss of generality we will assume that the decoherence basis coincide with the eigenvectors of $S_{z}=$ $V \sigma_{z} V^{\dagger}$ operator. The channel induced by a collision $U=|0\rangle\left\langle 0\left|\otimes V_{0}+\right| 1\right\rangle\langle 1| \otimes V_{1}$ reads

$$
\mathcal{E}=\left(\begin{array}{cccc}
1 & 0 & 0 & 0 \\
0 & \lambda \cos \varphi & \lambda \sin \varphi & 0 \\
0 & -\lambda \sin \varphi & \lambda \cos \varphi & 0 \\
0 & 0 & 0 & 1
\end{array}\right)
$$

where $\operatorname{tr}\left[V_{1}^{\dagger} V_{0} \xi\right]=\lambda e^{i \varphi}$. We can follow the same derivation of master equation as in the case of homogenization [4. However, for the illustration purposes we will describe the second procedure based on evaluation of $\log \mathcal{E}$. Unlike the case of homogenization the decoherence channel $\mathcal{E}$ defines a hermitian matrix, 
thus, the logarithm is pretty easy to calculate exploiting the simple functional calculus for hermitian operators.

Let us start with the observation that

$$
\lambda\left(\cos \varphi I+i \sin \varphi \sigma_{y}\right)=e^{\ln \lambda I} e^{i(\varphi+2 k \pi) \sigma_{y}}=e^{\ln \lambda I+i(\varphi+2 k \pi) \sigma_{y}},
$$

where $k$ is arbitrary natural number. For $k=0$ the logarithm is called principal. For our purposes it is sufficient to consider only this one, because we are restricted to angles inside the interval $[0,2 \pi]$. Since this matrix is the central part of the matrix $\mathcal{E}$, it follows that

$$
\mathcal{G}=\log \mathcal{E}=\left(\begin{array}{cccc}
0 & 0 & 0 & 0 \\
0 & \ln \lambda & -\varphi & 0 \\
0 & \varphi & \ln \lambda & 0 \\
0 & 0 & 0 & 0
\end{array}\right)
$$

is the generator of the semigroup dynamics containing the channel $\mathcal{E}$. Is $\mathcal{E}_{t}=e^{\mathcal{G} t}$ a valid quantum channel for any $t$ ?

Using the relations specified in Eqs.(96) we found that the only nonzero parameters are

$$
h_{3}=\varphi / 2, \quad c_{33}=-\ln \lambda / 2 .
$$

Since $c_{33} \geq 0$ it follows that the matrix $C$ is positive. Therefore

$$
\begin{aligned}
\mathcal{G}[\varrho] & =-i \frac{\varphi}{2 \hbar}\left[S_{z}, \varrho\right]-\frac{\ln \lambda}{4}\left(\left[S_{z}, \varrho S_{z}\right]+\left[S_{z} \varrho, S_{z}\right]\right) \\
& =-i \frac{\varphi}{2 \hbar}\left[S_{z}, \varrho\right]-\frac{\ln \lambda}{2}\left(S_{z} \varrho S_{z}-\varrho\right)
\end{aligned}
$$

defines a correct Lindblad generator. Using $H=\frac{1}{2} \varphi S_{z}$ and $\gamma=(2 \ln \lambda) / \varphi^{2}$ we obtain a well-known master equation

$$
\frac{d \varrho}{d t}=-\frac{i}{\hbar}[H, \varrho]-\frac{\gamma}{2}[H,[H, \varrho]],
$$

which is used to model the decoherence also for more dimensional systems.

\section{Conclusions}

In these lectures we introduced and investigated the simple collision model to capture relevant features and properties of quantum open system dynamics. We focused on two particular collision models determined by the choice of the unitary transformations describing the individual collisions:

- Homogenization induced by the partial swap interactions $U_{\eta}=\cos \eta I+$ $i \sin \eta S$. This process was motivated by the classical thermalization process (0th law of thermodynamics). 
- Decoherence induced by the controlled unitary interactions $U=\sum_{j}\left|\varphi_{j}\right\rangle\left\langle\varphi_{j}\right| \otimes$ $V_{j}$. This process describes the disappearance of the quantumness of quantum systems.

It is known that creation of entanglement requires interactions between quantum systems. In our collision model we start from completely factorized state. It is an interesting question what type of multipartite entanglement is created via a well-defined sequence of bipartite collisions. We haven't provided a definite answer to this problem, but we illustrated that the created entanglement of the particular collision models is not trivial. In particular, the partial swap interaction creates $W$-type of entanglement for which all the involved systems are pairwisely entangled although they did not interact directly. Moreover, during the whole evolution the CKW inequalities are saturated. The entanglement created in the decoherence collision model is of completely different quality. In this case the created entanglement is of $G H Z$-type meaning that pairwise entanglement in the reservoir vanishes, however the system is still entangled. The reservoir itself (the system is traced out) is in a separable state. The collision models can be understood as a (simple) preparation processes aiming to create multipartite entanglement. And it is of interest to understand what quality and quantity of entanglement it is capable of.

The well-defined sequence of collisions and specific initial conditions imply that the discrete time evolution of the system is described by a discrete semigroup of natural powers of $\mathcal{E}$. May this this discrete set of channels be interpolated by a single one-parametric continuum of channels? Is this continuum a semigroup of channels, or not? Luckily, we have derived that the interpolating continuum for both cases of homogenization and decoherences. By deriving the corresponding master equations and testing the Markovianity of the generator we showed that these sets form one-parametric semigroups of channels. Let us note that a collision model simulation of given semigroups of channels is easy. Each semigroup $\mathcal{E}_{t}$ can be discretized by introducing a parameter $\tau$ and set $\mathcal{E}_{n}=\mathcal{E}_{n \tau}$. Since the choice of an interaction $U$ inducing $\mathcal{E}_{\tau}$ is not unique, also the collision model is not unique. However, the particular collision dynamics of the system will be the same.

Let us note an interesting fact. Fix a unitary collision $U$. Even if $\xi, \xi^{\prime}$ induce a channels $\mathcal{E}, \mathcal{E}^{\prime}$ with valid generators $\mathcal{G}, \mathcal{G}^{\prime}$, the convex combination $\lambda \xi+(1-\lambda) \xi^{\prime}$ does not have to be associated with a correct Lindblad generator. There are collision models that cannot be interpolated by Markovian dynamics. Surprisingly, collision models provides richer dynamics than Lindblad's master equations. To sort out example consider Markovian models developed in order to describe true open system dynamics can be efficiently simulated (approximated) in collision models. We believe that such toy collision models provide an interesting playground capturing all the conceptual features of quantum open system dynamics. 


\section{Acknowledgments}

We would like to thank coauthors of the original papers Peter Štelmachovič, Valerio Scarani, Nicolas Gisin, and Mark Hillery. Many thanks also to Daniel Burgarth for inspiring discussions on closely related topics, which would deserve some space in these lectures, but due to space-time constraints finally left outside the scope. We acknowledge financial support of the European Union projects HIP FP7-ICT-2007-C-221889, and CE QUTE ITMS NFP 26240120009, and of the projects, CE SAS QUTE, and MSM0021622419.

\section{References}

[1] M.Ziman, P.Štelmachovič, V.Bužek, M.Hillery, V.Scarani, N.Gisin, Dilluting quantum information: An analysis of information transfer in systemreservoir interactions, Phys.Rev. A 65 , 042105 (2002).

[2] V.Scarani, M.Ziman, P.Štelmachovič, N.Gisin, V.Bužek, Thermalizing Quantum Machines: Dissipation and Entanglement, Phys.Rev.Lett. 88 , 97905-1 (2002).

[3] M.Ziman, P.Štelmachovič, V.Bužek, Description of quantum dynamics of open systems based on collision-like models, Open systems and information dynamics 12, No.1, pp. 81-91 (2005).

[4] M.Ziman, V.Bužek, All (qubit) decoherences: Complete characterization and physical implementations, Phys.Rev.A 72, 022110 (2005).

[5] E.Schrödinger, An Undulatory Theory of the Mechanics of Atoms and Molecules, Phys. Rev. 28, 10491070 (1926)

[6] E.B.Davies, Quantum Theory of Open Systems (Academic, London, 1976).

[7] R.Alicki, K.Lendi, Quantum Dynamical Semigroups and Applications, Lecture Notes in Physics (Springer-Verlag, Berlin, 1987).

[8] H.P.Breuer, The Theory Of Open Quantum Systems (Oxford University Press, USA, 2002).

[9] M.A.Nielsen, I.L.Chuang, Quantum Computation and Quantum Information (Cambridge University Press, Cambridge, 2000).

[10] T.Heinosaari, M.Ziman, Guide to mathematical concepts of quantum theory, Acta Physica Slovaca 58, 487-674 (2008).

[11] V. Bužek and M. Hillery, Quantum copying: Beyond the no-cloning theorem, Phys.Rev.A 54, 1844 (1996).

[12] V.Scarani, S.Iblisdir, N.Gisin, A.Acin, Quantum cloning, Rev.Mod.Phys. $77,1225-1256(2005)$ 
[13] M. Reed, B. Simon, Methods of Modern Mathematical Physics I: Functional Analysis (Academic Press, San Diego, 1980)

[14] W.H. Zurek, Decoherence, einselection, and the quantum origins of the classical, Rev.Mod.Phys. 75, 715 (2003).

[15] F.Buscemi, G.Chiribella, G.M.D'Ariano, Inverting quantum decoherence by classical feedback from the environment, Phys.Rev.Lett. 95, 090501 (2005).

[16] R.Horodecki, P.Horodecki, M.Horodecki, and K.Horodecki, Quantum entanglement, Rev.Mod.Phys. 81, pp. 865-942 (2009).

[17] M.B.Plenio, S.Virmani, An introduction to entanglement measures, Quant.Inf.Comp. 7, 1 (2007).

[18] W.K.Wootters, Entanglement of Formation of an Arbitrary State of Two Qubits, Phys.Rev.Lett. 80, 2245 (1998).

[19] V.Coffman, J.Kundu, W.K.Wootters, Distributed Entanglement, Phys.Rev.A 61, 052306 (2000).

[20] T.J.Osborne, F.Verstraete, General Monogamy Inequality for Bipartite Qubit Entanglement, Phys.Rev.Lett. 96, 220503 (2006).

[21] H. Spohn, Kinetic equations from Hamiltonian dynamics: Markovian limit, Rev.Mod.Phys. 53, 569 (1980).

[22] G. Lindblad, On the generators of quantum dynamical semigroups, Comm.Math.Phys. 48, pp. 119-130 (1976).

[23] M.M.Wolf, J.I.Cirac: Dividing quantum channels, Comm.Math.Phys. 279, pp. $147-168(2008)$

[24] L.V.Denisov, Infinitely divisible Markov mappings in the quantum theory of probability, Th. Prob. Appl. 33, 392 (1988) 